\title{
Aprendizaje Basado en Problemas como estrategia didáctica para el desarrollo del razonamiento lógico matemático
}

\section{Problem-Based Learning as a didactic strategy for the development of mathematical logical reasoning}

\author{
Jessica Johanna Tapia-Vélez \\ jessica.tapia@psg.ucacue.edu.ec \\ Universidad Católica de Cuenca, Azogues \\ Ecuador \\ https://orcid.org/0000-0002-6812-7230 \\ Darwin Gabriel Garcia-Herrera \\ dggarciah@ucacue.edu.ec \\ Universidad Católica de Cuenca, Azogues \\ Ecuador \\ https://orcid.org/0000-0001-6813-8100 \\ Juan Carlos Erazo-Álvarez \\ jcerazo@ucacue.edu.ec \\ Universidad Católica de Cuenca, Cuenca \\ Ecuador \\ https://orcid.org/0000-0001-6480-2270 \\ Cecilia Ivonne Narváez-Zurita \\ inarvaez@ucacue.edu.ec \\ Universidad Católica de Cuenca, Cuenca \\ Ecuador \\ https://orcid.org/0000-0002-7437-9880
}

Recibido: 25 de abril de 2020

Revisado: 30 de mayo de 2020

Aprobado: 15 de junio de 2020

Publicado: 30 de junio de 2020 


\title{
RESUMEN
}

La presente investigación tiene como propósito concienciar a los educadores sobre la relevancia del Aprendizaje Basado en Problemas (ABP) en el proceso de enseñanzaaprendizaje en la asignatura de matemáticas; esto fomenta el desarrollo de habilidades y pensamientos lógicos, teniendo presente los conocimientos previos y problemas vivenciales consiguiendo que los alumnos comprendan los conceptos y actividades prácticas de forma relacional. Metodológicamente se fundamentó desde una investigación tipo descriptiva correlacional no experimental transversal, para la recolección de información se empleó la encuesta y un cuestionario elaboradas en escala Likert, aplicada en un único momento. Con los resultados obtenidos se confirma el objetivo de la investigación; mismo que, propone la aplicación del ABP como estrategia didáctica para el desarrollo del razonamiento lógico matemático en los estudiantes, motivando y potenciando de una manera efectiva y rápida la resolución de problemas con diferentes alternativas y como nuevo método de aprendizaje de las matemáticas.

Descriptores: Experimento educacional; investigación pedagógica; matemáticas; aprendizaje activo. (Palabras tomadas del Tesauro UNESCO).

\begin{abstract}
The purpose of this research is to educate educators on the relevance of Problem Based Learning $(\mathrm{ABP})$ in the teaching-learning process in the subject of mathematics; This encourages the development of logical thoughts and abilities, taking into account previous knowledge and experiential problems, making students understand relational concepts and practical activities. Methodologically it was based on a cross-sectional nonexperimental descriptive correlational type of research, for the collection of information the survey and a questionnaire prepared on a Likert scale, applied in a single moment, were used. With the results obtained, the objective of the investigation is confirmed; It also proposes the application of ABP as a didactic strategy for the development of mathematical logical reasoning in students, effectively and quickly motivating and enhancing problem solving with different alternatives and as a new method of learning mathematics.
\end{abstract}

Descriptors: Educational experiments; Educational research; Mathematics; Activity learning. (Words taken from the UNESCO Thesaurus). 


\section{INTRODUCCIÓN}

En el proceso de enseñanza aprendizaje de la matemática se ha encontrado conflictos en los docentes y alumnos, dando mayor importancia a los contenidos establecidos en el currículo vigente, olvidando activar habilidades como: entender, razonar y resolver; es necesario, recalcar que, los estudiantes están siendo repositorio de conocimientos dejando de buscar soluciones óptimas a un problema planteado. La importancia de las matemáticas a nivel mundial ocupa un lugar muy importante y transcendental en los sistemas educativos, tiene un constante crecimiento e innovación porque todo lo que está en el entorno se relaciona con ella; por esta razón, se enfatiza en los diferentes métodos de enseñanza y aprendizaje para que los estudiantes adquieran nuevos conocimientos y habilidades.

Hoy en día, el Ecuador enfrenta deficiencias en razonamiento lógico y resolución de problemas matemáticos, esta situación ha sido afirmada con los resultados obtenidos en PISA (Programa para la Evaluación Internacional de los Alumnos) 2018; en consecuencia, se infiere que como país tenemos una gran debilidad en educación, formamos estudiantes mecánicos y no permitimos que desarrollen su creatividad y presunción a la resolución de problemas de una forma lógica, rápida y eficaz.

Abordando los problemas anteriores, en las Instituciones Educativas a nivel de la Zona 6 Azuay, Cañar y Morona Santiago, especialmente en el cantón de Biblián se ha observado un bajo aprovechamiento en el área de matemáticas llegando a varias conclusiones que pueden ser predominantes: la asignatura es considerada por los estudiantes la más difícil, denominada en la jerga juvenil el "cuco de las materias", la carencia de preparación en el uso de estrategias didácticas hace que exista la desmotivación y el desinterés por parte del estudiante llegando a un tradicionalismo que consiste en que el docente siempre tenga la razón convirtiéndose en clases monótonas; además, varios docentes no cumplen con los perfiles adecuados para impartir dicha asignatura. 
Como lo afirma (Leiva-Sánchez, 2016), los maestros deben tener vocación en el área de matemáticas para no instituir deficiencias en el desarrollo del razonamiento lógico; así también implementar, metodologías activas que permitan al alumno ser dinámico y actor principal de la construcción de su conocimiento para llegar a un aprendizaje significativo. De lo expuesto anteriormente el docente para construir un aprendizaje en los estudiantes, debe capacitarse de manera continua y permanente sobre las diferentes metodologías activas existentes, así crear un ambiente dinámico y motivador, potenciando el desarrollo del pensamiento lógico matemático que ayude en la toma de decisiones para la resolución de un problema o ejercicio (Saltos-Cedeño, et al., 2020).

Por lo descrito anteriormente, la presente investigación propone la aplicación de una estrategia didáctica como es el Aprendizaje Basado en Problemas ABP para el desarrollo del pensamiento lógico matemático a los 157 docentes que imparten la asignatura de matemáticas en 21 establecimientos del cantón Biblián, sin descuidar el objetivo de la planificación en el aula. El ABP permite a los estudiantes resolver problemas de la vida cotidiana, buscando diferentes alternativas a ser aplicadas para llegar a una solución, generando un rol activo en su aprendizaje que le permita desarrollar cooperación, responsabilidad, habilidades y actitudes con recursos que beneficiaran de forma personal y académica.

\section{Referencial teórico}

Existen varias indagaciones enfocadas a la metodología del Aprendizaje Basados en Problemas aplicadas en el proceso de enseñanza-aprendizaje, a continuación, se presentan varias investigaciones: Según, (Valderrama-Sanabria \& Castaño-Riobueno, 2017) la investigación se realizó en la Universidad de Santander en Bucaramanga, Colombia tiene como objetivo: destacar el ABP como estrategia metodológica para alcanzar un aprendizaje eficaz y perdurable, que permita solucionar situaciones reales. Para verificar su objetivo planteado aplico el método descriptivo y los resultados confirman que la aplicación del ABP fortalece el pensamiento crítico y reflexivo, generado la autonomía del aprendizaje y el razonamiento lógico en el alumno. 
Además (Cedeño-Loor, 2017), señala sobre su investigación aplicada en la Universidad de Manabí ubicada en Ecuador, en la que aborda la relación que existe entre la asignatura de matemáticas y la resolución de problemas contextualizados, exalta la importancia de una metodología como es ABP para el aprendizaje en la resolución de problemas utilizando un enfoque cuantitativo a través de instrumentos como son un pretest y postest los cuales se aplicaron a un grupo de control y un grupo experimental; además, el grupo experimental recibió capacitaciones en base al procedimiento heurístico de Polya y los resultados obtenidos fueron significativos en el aprendizaje lógico de forma razonable y critica.

El último artículo mencionado hace referencia a (Paredes-Curin, 2016) quien analizo el proceso de la fotosíntesis mediante la metodología de un aprendizaje basado en problemas con un enfoque cualitativo y una encuesta como instrumento y fichas de observación, la metodología aplicada es descriptiva. El resultado obtenido es la un autoaprendizaje correcto y significativo y la aplicación de la metodología Aprendizaje Basados en Problemas reflexionar sobre los investigado por los estudiantes de manera que permite resolver problemas de forma adecuada para refutar los resultados.

Con los artículos antes mencionados se observa aplicación la metodología del ABP es muy significativa para la construcción del conocimiento lógico generando un aprendizaje dinámico, con razonamiento crítico y reflexivo en los estudiantes motivado para la investigación de estrategias y resultados de cualquier problema enunciado. E pensamiento lógico es la facultad que tiene el ser humano desde una edad temprana, iniciando con comparaciones entre elementos, siguiendo con la clasificación por su forma o tamaño y así sucesivamente. Como refiere (Jaramillo-Naranjo \& Puga-Peña, 2016), a medida que vamos creciendo desarrollamos un pensamiento lógico y generamos criterios, reflexiones, análisis y conclusiones para resolver problemas en el entorno vivencial, creando un proceso de enseñanza.

Para generar un pensamiento lógico, se debe considerar el nivel de conocimiento que poseen los estudiantes para la aplicación de metodologías, actividades y dinámicas. Por este motivo el presente trabajo indica las etapas del desarrollo del conocimiento descritas 
por Piaget las mismas que se relacionan con la práctica docente dentro del aula para elaborar y aplicar diferentes metodologías y estrategias que produzcan un pensamiento lógico para tomar decisiones acertadas y eficaces. (Piaget, 1991) afirma que, el desarrollo del conocimiento del niño es un proceso en el que intervienen la maduración biológica y su relación con el entorno, adicionalmente manifiesta que existen 4 etapas del desarrollo del conocimiento mismas que se encuentran definidas de la siguiente manera:

1. Sensorio motor (nacimiento - 2 años de edad). - Concibe su conocimiento a través de la interacción física sensorial y el uso de objetos hasta cuando empieza hablar, captando de una mejor manera la información por medio de sus sentidos expresando premisa cortas y sencillas, además son capaces de relacionar la imagen con su respectivo nombre.

2. Pre-operacional (2 - 7 años). - En esta etapa predominan los juegos de imitación, todavía no posee una lógica concreta, comprende e interpreta signos y símbolos y aflora el egocentrismo.

3. Operaciones Concretas $(7-11$ años). - Comienza con sus primeras manifestaciones de pensamiento lógico, son menos egocéntricos, esto quiere decir que son capaces de ponerse en la situación del otro individuo y también expresan sentimientos, pensamientos y opiniones.

4. Operaciones Formales (12 años - edad adulta). - Es la etapa final, incrementa la lógica de la cual genera la capacidad de utilizar un razonamiento abstracto y además utiliza el razonamiento hipotético deductivo.

Lo descrito anteriormente se deduce que el niño desde su nacimiento va desarrollando su conocimiento acorde a su etapa de vida hasta llegar a tener un conocimiento cognitivo el cual le permite crear hipótesis y posteriormente dar soluciones. El razonamiento lógico en los estudiantes es muy escaso esto se debe a que utilizan patrones memorísticos y no analizan el problema propuesto que requiere una solución.

Por lo antes mencionado es muy importante desarrollar el razonamiento lógico matemático en los estudiantes para construir conocimientos que ayuden a la formación de habilidades como entender, calcular premisas y nociones para crear relaciones 
lógicas. Como menciona (Larrazolo, Backhoff, \& Tirado, 2013) la enseñanza de manera analítica, comparativa, valorativa y reflexiva, es esencial para que el alumno sea capaz de resolver cualquier problema, mediante las cuales generan un aporte muy importante en lo referente al aprendizaje y la construcción del conocimiento.

Como lo afirma (Navarro-Casabuena, 2017) el razonamiento lógico matemático es importante para la resolución de problemas que poseen los individuos para poder llegar a soluciones con una particularidad, de ser un proceso sistematizado en unir premisas de una forma lógica encaminadas a llegar a una conclusión. El razonamiento lógico matemático se debe ejercitar diariamente mediante el uso de recursos adecuados que permitan resaltar la capacidad de comparar y analizar problemas para llegar a conclusiones fundamentadas y sólidas que luego sean aplicadas.

Por lo expuesto anteriormente el docente debe incentivar al estudiante a crear su propio conocimiento con actividades pertinentes que provoquen la necesidad de concebir esos conocimientos. Cuando el estudiante empieza a razonar sobre problemas planteados crea un razonamiento lógico que es muy importante, y de esta manera se forma un alumno constructivo que no va aplicar procedimientos logarítmicos, sino que, va a crear otras vías para resolver problemas y así refleja la capacidad de entendimiento y un aprendizaje que perdura.

En el siglo XX los modelos de aprendizaje están cambiando, ha pasado de un modelo conductista que se basa en la repetición de contenidos a un modelo constructivista que conduce, analiza, expone y resuelve problemas hipotéticos y reales a partir de un trabajo investigativo y colaborativo entre estudiantes (Matamorros-Espinoza, 2018).

Una de las técnicas didácticas más utilizadas para resaltar el constructivismo es el Aprendizaje Basado en Problemas $[A B P]$, su base es la resolución de problemas frecuentes de manera colaborativa en equipos pequeños, permitiendo desarrollar en el estudiante la capacidad de analizar, razonar, valorar, reflexionar, criticar, etc. en diferentes temáticas (Guevara-Mora, 2010). Cabe mencionar que ABP tiene como objetivo la relación de lo teórico con lo práctico, poniendo énfasis en desarrollo del pensamiento lógico. Los problemas planteados pueden llegar a ser un reto en cada una 
de las asignaturas; los escenarios son simulaciones de la problemas vivenciales y fantásticos que involucran la resolución de cada uno de ellos como lo afirman (Vargas \& Labatut-Portilho, 2017).

Además, rol del docente en la aplicación del ABP es muy importante ya que su actuación es el de ser un tutor que ayudara a sus estudiantes a identificar, reflexionar sobre la información propuesta, motivando a conseguir de una forma adecuada el objetivo planteado sin desviarse del camino, además orientara a identificar situaciones importantes que conlleven a la resolución del problema de manera personal y grupal.

Como expone (Nasution, Yerizon, \& Gusmiyanti, 2018) lo descrito anteriormente se alcanza elaborando por parte del docente preguntas generalizadas que faciliten al estudiante comprender el problema planteado manteniendo el interés en conseguir información precisa de una manera adecuada para buscar diversas alternativas para la creación y ejecución de un plan que se realizara de manera sistemática para la resolución del problema y sus resultados serán expuesto con criterios reflexivos si fue la mejor solución. Es muy importante señalar a (Polya, 1965) establece que existe 4 etapas para la aplicación del aprendizaje basado en problemas:

1. Entender el problema: Consiste en que los estudiantes entiendan el problema en sus propias palabras diferenciando datos e incógnitas y determinen a donde se va a llegar, es decir reformular el problema con lenguaje entendible sin cambiar el contexto.

2. Elaboración de plan: Crear estrategias adecuadas mediante la investigación que nos lleve a la resolución de un determinado problema, el rol del docente es el de ser un guía.

3. Ejecutar el plan: Implementar las estrategias antes mencionadas para llegar a la solución efectiva del problema verificando cada uno de los pasos con claridad.

4. Analizar los resultados: Verificar los resultados obtenidos y analizarlos si son acordes con el problema planteado inicialmente, en esta etapa se consolida el conocimiento. 


\section{MÉTODO}

Metodológicamente se fundamentó desde una investigación tipo descriptiva correlacional no experimental transversal, para la recolección de información se empleó la encuesta y un cuestionario de 7 preguntas elaboradas en escala Likert, aplicada en un único momento (Mendoza-Novillo, Erazo-Álvarez, \& Narváez-Zurita, 2019). El universo poblacional estuvo conformado por 31 Establecimientos Educativos del cantón Biblián con 221 docentes, del cual, se aplicó un muestreo aleatorio estratificado tomando como base a un grupo de 157 docentes que imparten la asignatura de matemáticas; además, sobre la base de lo descrito anteriormente para determinar el tamaño de la muestra se empleó una formula con el $99 \%$ de fiabilidad y $5 \%$ de margen de error, dando como resultado que deberían ser 128 los educadores encuestados.

Para validar la encuesta mediante el coeficiente de Alfa de Cronbach mismo que arrojó un resultado de 0,703 que significa que la encuesta aplicada es confiable para su aplicación. Finalmente se realizó un análisis estadístico de los datos recopilados en el software SPSS para la presentación de los resultados de la investigación (Ávila-Sacoto, Erazo-Álvarez, Narváez-Zurita, \& Erazo-Álvarez, 2019).

\section{RESULTADOS}

En este apartado se proporcionará los principales resultados obtenidos a las preguntas planteadas en el proceso de investigación de la encuesta aplicada a los docentes que imparten la materia de matemáticas en los diferentes niveles de Educación Inicial hasta Bachillerato General Unificado de las instituciones Educativas del Cantón Biblián; posteriormente la información obtenida se analizará a través de los siguientes gráficos y tablas. 


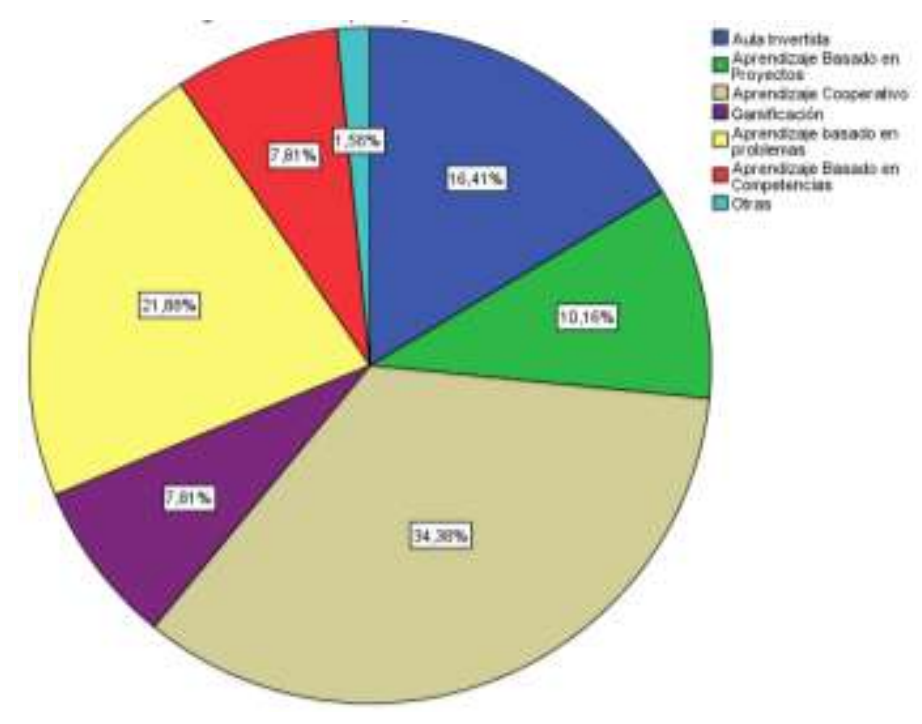

Figura 1. Diversas Metodologías activas aplicadas dentro de clases de matemáticas. Fuente: Elaboración propia

Como se puede observar en el Figura 1, y tomando en cuenta los 3 porcentajes más significativos, del total de los 128 docentes encuestados el 34,38\% que corresponden a 44 afirman que durante la clase de matemáticas aplican el Aprendizaje Colaborativo; el $21.88 \%$ que corresponde a 28 educadores aplican el aprendizaje basado en problemas; el 16,41\% que corresponde a 21 maestros aplican la metodología del aula invertida y en porcentajes menores el aprendizaje basados en proyectos, Aprendizaje Basado en Competencias, Gamificación y otras. En consecuencia, por lo expuesto se verifica que los educadores utilizan diferentes metodologías activas dentro del aula de clases. 
Revista Arbitrada Interdisciplinaria KOINONIA

Año 2020. Vol V. ํ⒈ Especial Educación

Hecho el depósito de Ley: FA2016000010

ISSN: 2542-3088

FUNDACIÓN KOINONIA (F.K). Santa Ana de Coro. Venezuela.

Jessica Johanna Tapia-Vélez; Darwin Gabriel Garcia-Herrera; Juan Carlos Erazo-Álvarez; Cecilia Ivonne Narváez-Zurita

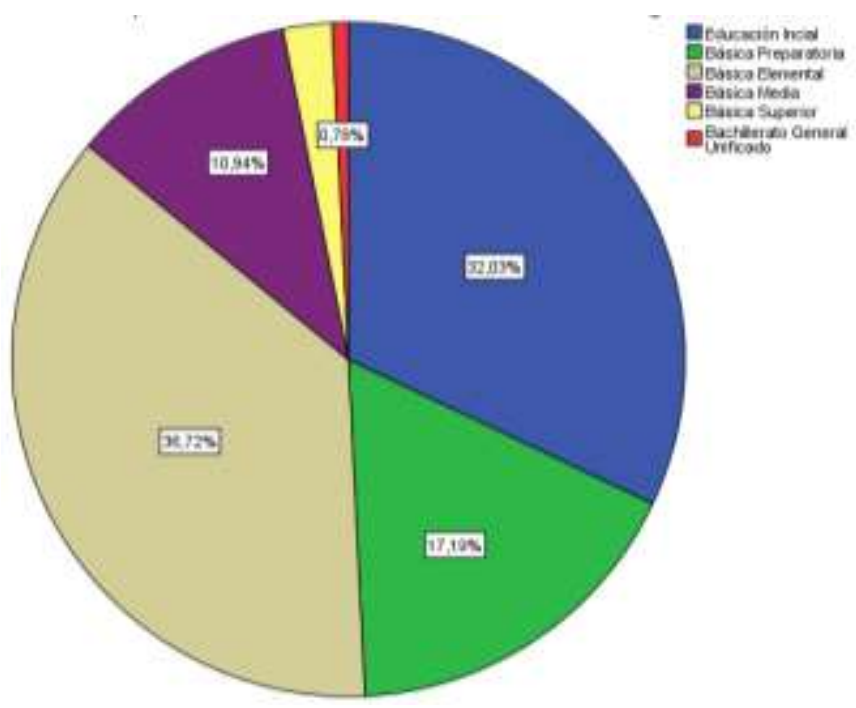

Figura 2. Falta de razonamiento lógico matemático. Fuente: Elaboración propia

En el Figura 2, se evidencia que de los docentes encuestados el 36,72\% que corresponde al nivel elemental y 32,03 que corresponde a la Educación Inicial afirman que los estudiantes tienen falencias en el razonamiento lógico matemático.

\section{Tabla 1}

En la planificación de matemáticas está presente el ABP

\begin{tabular}{cccccc} 
& & Frecuencia & Porcentaje & Porcentaje válido & $\begin{array}{c}\text { Porcentaje } \\
\text { acumulado }\end{array}$ \\
\hline \multirow{4}{*}{ Válido } & & & & & 23,4 \\
& Nunca & 30 & 23,4 & 23,4 & 45,3 \\
& Casi nunca & 28 & 21,9 & 21,9 & 78,9 \\
& Ocasionalmente & 43 & 33,6 & 33,6 & 80,5 \\
& Casi siempre & 2 & 1,6 & 1,6 & 100,0 \\
& Siempre & 25 & 19,5 & 19,5 &
\end{tabular}

Fuente: Elaboración propia

En la Tabla 1 y tomando como referencia el porcentaje acumulado se evidencia que el $78,9 \%$ de los docentes nunca, casi nunca y ocasionalmente aplican el Aprendizaje Basado en Problemas en sus planificaciones en la asignatura de matemáticas, siendo 
Revista Arbitrada Interdisciplinaria KOINONIA

Año 2020. Vol V. ํ⒈ Especial Educación

Hecho el depósito de Ley: FA2016000010

ISSN: $2542-3088$

FUNDACIÓN KOINONIA (F.K). Santa Ana de Coro. Venezuela.

Jessica Johanna Tapia-Vélez; Darwin Gabriel Garcia-Herrera; Juan Carlos Erazo-Álvarez; Cecilia Ivonne Narváez-Zurita

únicamente el $21,1 \%$ del total los docentes encuestados los que aplican siempre y casi siempre el ABP.

\section{Tabla 2}

EI ABP fortalece el razonamiento lógico matemático en los estudiantes*Aplica el ABP en clases de matemáticas tabulación cruzada

\begin{tabular}{|c|c|c|c|c|c|c|c|}
\hline & & \multicolumn{5}{|c|}{ Aplica el ABP en clases de matemáticas } & \multirow[t]{2}{*}{ Total } \\
\hline & & Nunca & $\begin{array}{l}\text { Casi } \\
\text { nunca }\end{array}$ & Ocasionalmente & $\begin{array}{c}\text { Casi } \\
\text { siempre }\end{array}$ & Siempre & \\
\hline \multirow{5}{*}{$\begin{array}{l}\text { El ABP fortalece el } \\
\text { razonamiento lógico } \\
\text { matemático en los } \\
\text { estudiantes }\end{array}$} & $\begin{array}{c}\text { Totalmente en } \\
\text { desacuerdo }\end{array}$ & 10 & 2 & 8 & 0 & 0 & 20 \\
\hline & En desacuerdo & 10 & 19 & 11 & 2 & 0 & 42 \\
\hline & $\begin{array}{c}\text { Ni de acuerdo ni en } \\
\text { desacuerdo }\end{array}$ & 1 & 12 & 22 & 1 & 1 & 37 \\
\hline & De acuerdo & 0 & 1 & 0 & 1 & 0 & 2 \\
\hline & $\begin{array}{l}\text { Totalmente de } \\
\text { acuerdo }\end{array}$ & 0 & 3 & 1 & 1 & 22 & 27 \\
\hline \multicolumn{2}{|c|}{ Total } & 21 & 37 & 42 & 5 & 23 & 128 \\
\hline
\end{tabular}

Pruebas de chi-cuadrado

$\begin{array}{cccc} & \text { Valor } & \text { gl } & \text { Sig. asintótica (2 caras) } \\ \text { Chi-cuadrado de Pearson } & 139,229^{\mathrm{a}} & 16 & , 000 \\ \text { Razón de verosimilitud } & 124,804 & 16 & , 000 \\ \text { Asociación lineal por lineal } & 67,102 & 1 & , 000 \\ \text { N de casos válidos } & 128 & & \end{array}$

a. 13 casillas $(52,0 \%)$ han esperado un recuento menor que 5 . El recuento mínimo esperado es ,08.

\section{Fuente: Elaboración propia}

En la tabla 2 se realizó una tabla cruzada entre el $A B P$ fortalece el razonamiento lógico matemático en los estudiantes versus la aplicación del ABP en la clase de matemáticas aplicando el chi-cuadrando, donde se obtuvo una significación asintótica menor que 0.5 lo que denota que la hipótesis es afirmativa y que existe una correlación entre las preguntas planteadas; por lo que, se deduce que si el docente aplica el ABP en las clases 
de matemáticas fortalece el razonamiento lógico; mientras que, por otra parte se infiere que si no aplica el $A B P$ no se fortalece el razonamiento lógico matemático en los estudiantes.

Para (Navarro-Casabuena, 2017) en su investigación sobre el desarrollo de un pensamiento matemático en el proceso de enseñanza aprendizaje en la asignatura de matemáticas que le permite al estudiante ser flexible, analítico y crítico. El Resultado fue exitoso para toma de decisiones de problemas propuestos creando muchas estrategias para llegar a soluciones de una manera rápida y eficaz, A demás, resalta el ambiente del aula que sea dinámico y motivador para el estudiante.

El Aprendizaje Basado en Problemas es muy importante para el proceso de enseñanzaaprendizaje del razonamiento lógico matemático; por esta razón, el docente debe estar preparado para ofrecer al estudiante una educación de calidad. De los resultados obtenidos en la presente investigación, se afirman que, el ABP no está presente dentro de la planificación de actividades en la asignatura de matemáticas, en consecuencia, tampoco el docente aplica esta metodología al impartir las clases de matemáticas lo cual no ayudara a fomentar el razonamiento lógico.

Con lo descrito anteriormente se propone la aplicación del ABP como estrategia didáctica para el desarrollo del razonamiento lógico matemático en los docentes que imparten la asignatura de matemáticas en los 21 Establecimientos Educativos del cantón Biblián para que de esta manera permita motivar a los estudiante a poseer un razonamiento crítico y reflexivo provocando que la materia sea de fácil entendimiento desde los primeros niveles de la educación básica hasta cuando se desarrolle en la vida profesional. 


\section{PROPUESTA}

La capacitación propuesta sobre la Aplicación del aprendizaje basado en problemas como estrategia didáctica para fortalecer el razonamiento lógico matemático está planeada para ser impartida en 2 sesiones.

La Forma del trabajo se ha estructurado a través de 4 ejes fundamentales que son la introducción, el desarrollo, la ejecución y la evaluación. La aplicación se basa en que los integrantes del grupo escuchan las exposiciones orales, posteriormente proceden a juntarse en pequeños grupos, analizan la información mediante técnicas de aprendizaje grupal y la ponen en práctica. El facilitador guiará en las actividades a ser planteadas y se sugiere un tiempo para cada actividad.

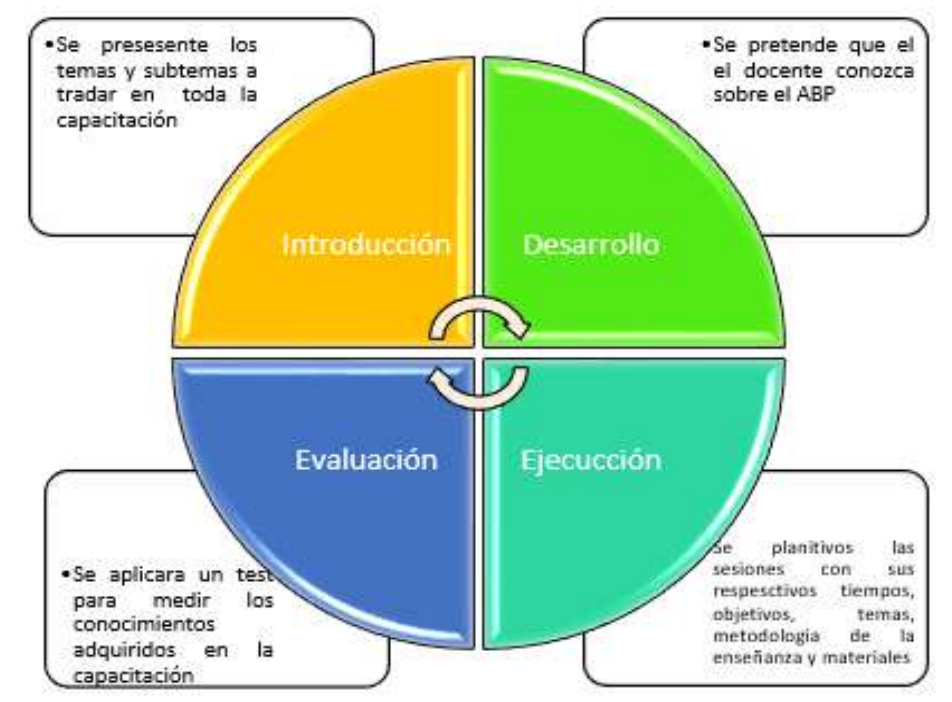

Figura 3. Estructura de la capacitación sobre la Aplicación del aprendizaje basado en problemas como estrategia didáctica para fortalecer el razonamiento lógico matemático.

Introducción. En este taller se mostrará el concepto del pensamiento lógico sus fases y el razonamiento lógico además se abordará la conceptualización del ABP, objetivos y características, un análisis comparativo de las metodologías tradicionales con el ABP para finalmente exponer todos los pasos que se debe seguir de forma sistemática y explicativa dentro de la aplicación del ABP con sus principales elementos y de esta manera fortalecer el razonamiento lógico de los estudiantes. 
Desarrollo. Durante la capacitación se pretende que el docente sea capaz de entender y aplicar de una manera efectiva el ABP como estrategia didáctica para el desarrollo del razonamiento lógico matemático.

Ejecución. Para la ejecución de las sesiones que se han planificado en el presente trabajo se consideran tablas descriptivas que se desarrollarán posteriormente; mismas que, tratan de englobar de una manera objetiva y sin descuidar el aspecto pedagógico la aplicación del ABP

\section{Tabla 3}

Planificación de la sesión 1

\section{Sesión 1}

Objetivo: Analizar el razonamiento lógico y los aspectos relevantes del ABP mediante técnicas de aprendizajes grupales para fortalecer las prácticas docentes dentro del aula.

\begin{tabular}{|c|c|c|c|c|}
\hline Tiempo & Objetivo & Temas o subtemas & $\begin{array}{l}\text { Metodología de la } \\
\text { enseñanza }\end{array}$ & Materiales \\
\hline $10 \min$ & $\begin{array}{l}\text { Conocer al } \\
\text { facilitador, la } \\
\text { dinámica de trabajo } \\
\text { y los objetivos de la } \\
\text { capacitación }\end{array}$ & $\begin{array}{l}\text { Bienvenida } \\
\text { Presentación de } \\
\text { los facilitadores } \\
\text { Reglas }\end{array}$ & $\begin{array}{l}\text { Bienvenida } \\
\text { Presentación de } \\
\text { los facilitadores } \\
\text { Reglas }\end{array}$ & $\begin{array}{l}\text { Pizarra } \\
\text { Proyector } \\
\text { Computador } \\
\text { Marcadores } \\
\text { Esferos } \\
\text { Lápices }\end{array}$ \\
\hline $15 \min$ & $\begin{array}{l}\text { Conocer a los } \\
\text { miembros del grupo } \\
\text { e identificar miedos } \\
\text { y expectativas } \\
\text { respecto a la capacitación }\end{array}$ & $\begin{array}{l}\text { Presentación de } \\
\text { los integrantes del } \\
\text { grupo } \\
\text { Expectativas } \\
\text { respecto al curso }\end{array}$ & $\begin{array}{l}\text { Dinámica de } \\
\text { presentación }\end{array}$ & $\begin{array}{l}\text { Identificadores } \\
\text { Lápices } \\
\text { Papeles }\end{array}$ \\
\hline $30 \mathrm{~min}$ & $\begin{array}{l}\text { Conceptualizar } \\
\text { pensamiento lógico }\end{array}$ & $\begin{array}{l}\text { ¿Qué es el } \\
\text { pensamiento lógico? }\end{array}$ & $\begin{array}{l}\text { Lluvia de idea } \\
\text { Exposición oral } \\
\text { Del concepto }\end{array}$ & $\begin{array}{l}\text { Pizarra } \\
\text { marcadores } \\
\text { Computadora } \\
\text { proyector } \\
\text { Presentación } \\
\text { visual }\end{array}$ \\
\hline $30 \mathrm{~min}$ & $\begin{array}{l}\text { Identificar las fases del } \\
\text { pensamiento lógico }\end{array}$ & $\begin{array}{l}\text { ¿Cuáles son las fases } \\
\text { del pensamiento } \\
\text { lógico? }\end{array}$ & $\begin{array}{l}\text { Exposición oral } \\
\text { Juego de roles }\end{array}$ & $\begin{array}{l}\text { Computadora } \\
\text { proyector } \\
\text { Presentación } \\
\text { visual }\end{array}$ \\
\hline $30 \min$ & $\begin{array}{l}\text { Conceptualizar } \\
\text { razonamiento lógico }\end{array}$ & $\begin{array}{l}\text { ¿Qué es el } \\
\text { razonamiento lógico? }\end{array}$ & $\begin{array}{l}\text { Lluvia de idea } \\
\text { Exposición oral } \\
\text { Del concepto }\end{array}$ & $\begin{array}{l}\text { Pizarra } \\
\text { marcadores } \\
\text { Computadora }\end{array}$ \\
\hline
\end{tabular}


Revista Arbitrada Interdisciplinaria KOINONIA

Año 2020. Vol V. ํ⒈ Especial Educación

Hecho el depósito de Ley: FA2016000010

ISSN: $2542-3088$

FUNDACIÓN KOINONIA (F.K). Santa Ana de Coro. Venezuela.

Jessica Johanna Tapia-Vélez; Darwin Gabriel Garcia-Herrera; Juan Carlos Erazo-Álvarez; Cecilia Ivonne Narváez-Zurita

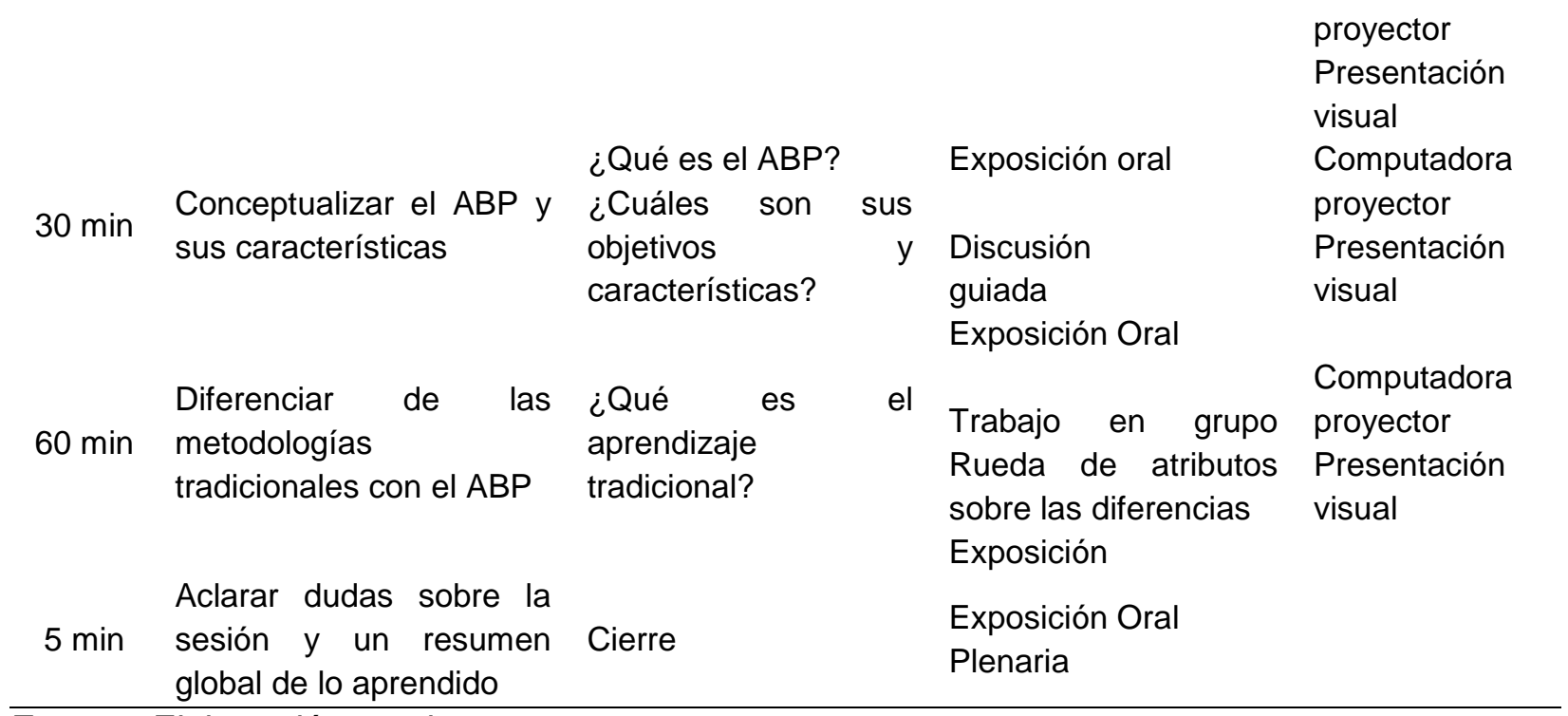

Fuente: Elaboración propia

\section{Tabla 4}

Planificación de la sesión 2.

\section{Sesión 2}

Objetivo Describir el proceso del ABP mediante problemas matemáticos para fomentar el razonamiento lógico

\begin{tabular}{|c|c|c|c|c|}
\hline Tiempo & Objetivo & Temas o subtemas & $\begin{array}{c}\text { Metodología de } \\
\text { la enseñanza }\end{array}$ & Materiales \\
\hline $30 \mathrm{~min}$ & $\begin{array}{c}\text { Establecer el proceso } \\
\text { del ABP }\end{array}$ & $\begin{array}{l}\text { ¿Según Polya (1965) cuáles } \\
\text { son las etapas de ABP para } \\
\text { la resolución de Problemas? }\end{array}$ & $\begin{array}{l}\text { Exposición oral } \\
\text { Discusión } \\
\text { guiada }\end{array}$ & $\begin{array}{l}\text { Computadora } \\
\text { proyector } \\
\text { Presentación } \\
\text { visual }\end{array}$ \\
\hline $60 \mathrm{~min}$ & $\begin{array}{c}\text { Establecer cuáles son } \\
\text { los elementos básicos } \\
\text { que intervienen en el } \\
\text { ABP }\end{array}$ & $\begin{array}{c}\text { ¿Cuál es el papel del tutor? } \\
\text { ¿Cuál es el papel del } \\
\text { estudiante? } \\
\text { ¿Cómo se interactúa en } \\
\text { grupos? }\end{array}$ & $\begin{array}{c}\text { Rejilla } \\
\text { Plenaria }\end{array}$ & $\begin{array}{l}\text { Documento } \\
\text { Lápices } \\
\text { Papel }\end{array}$ \\
\hline $90 \mathrm{~min}$ & $\begin{array}{c}\text { Fomentar el ABP a } \\
\text { través de la resolución } \\
\text { de problemas }\end{array}$ & $\begin{array}{c}\text { Implementación paso a paso } \\
\text { en Propuesta de situaciones } \\
\text { problemáticas }\end{array}$ & $\begin{array}{l}\text { Exposición } \\
\text { Oral } \\
\text { Discusión } \\
\text { Guiada } \\
\text { El proyecto } \\
\text { Exposición } \\
\text { grupal }\end{array}$ & $\begin{array}{l}\text { Computadora } \\
\text { proyector } \\
\text { Presentación } \\
\text { visual } \\
\text { Documento } \\
\text { Lápices } \\
\text { Papel }\end{array}$ \\
\hline
\end{tabular}

Fuente: Elaboración propia 
Evaluación: En esta sección se aclararán las interrogantes derivadas del proceso de capacitación de una forma global iniciando con una discusión guiada sobre si se cumplieron las expectativas que tuvieron al inicio de la capacitación, pasando por una ronda de preguntas y respuestas para aclarar posibles dudas de la sesión mediante una exposición oral para finalmente proceder con la aplicación de un test que permitirá evaluar el conocimiento adquirido de todos los temas y subtemas plantados en la planificación.

\section{CONCLUSIONES}

Del análisis de la información descrita en el presente trabajo se puede inferir que:

Con el ABP es posible cambiar de una estrategia tradicional donde el aprendizaje de la información es repetitivo y mecánico a estrategias constructivista, donde se crea, se transfiere y se produce la abstracción de los contenidos que le permitirán al estudiante tener un aprendizaje perdurable.

La aplicación del ABP en la asignatura de matemáticas tiene mucha importancia; puesto que, genera en los estudiantes la capacidad de crear varias estrategias para la solución de un problema, desarrollando de esta manera el pensamiento lógico, crítico y reflexivo para una mejor compresión.

Para esta investigación se logró realizar un diagnóstico a los docentes que imparte las clases de matemáticas en los 21 establecimientos del cantón Biblián sobre la presencia y la aplicación de la metodología activa del ABP; para lo cual, se seleccionó un muestreo estratificado, cuyos resultados permitieron afirmar la necesidad de aplicar el tema de investigación.

De acuerdo al muestreo realizado se puede verificar que las falencias detectadas en Educación Inicial y Básica Media, se dan debido a que no se fortalece el aprendizaje de las matemáticas

No se evidencia la presencia del $\mathrm{ABP}$ en las planificaciones en la asignatura de matemáticas; es por ello que, se puede señalar que al desarrollar un problema planteado no siguen la secuencia adecuada para llegar a la solución. 
El presente trabajo propone crear un bosquejo de planificación para el aprendizaje del $\mathrm{ABP}$ en los docentes que imparten la asignatura de matemáticas que permita cubrir en su totalidad el proceso de aprendizaje, incluyendo actividades de aplicación en problemas matemáticos mediante la elección de los objetivos y técnicas grupales para desarrollar competencia en dicha metodología.

\section{FINANCIAMIENTO}

No monetario.

\section{AGRADECIMIENTO}

A los docentes que imparten la materia de matemáticas en los diferentes niveles de Educación Inicial hasta Bachillerato General Unificado de las instituciones Educativas del Cantón Biblián; por su apoyo en el desarrollo de la investigación.

\section{REFERENCIAS CONSULTADAS}

Ávila-Sacoto, E., Erazo-Álvarez, J. C., Narváez-Zurita, C. I., \& Erazo-Álvarez, C. A. (2019). Estrategias de marketing digital 2.0 para la generación de ingresos en Pymes de servicios. [Digital Marketing 2.0 strategies for generating income in service Pymes] Recuperado de: https://n9.cl/yz442. Cienciamatria, 187-214. doi:https://doi.org/10.35381/cm.v5i1.264

Cedeño-Loor, F. (2017). Importancia del método de resolución de problemas con ejemplo de la vida diaria en el aprendizaje de matemática en los estudiantes del nivel I de la Universidad Técnica de Manabí - Ecuador, 2015 [Importance of the method of solving problems with example ]. Lima: Universidad Nacional Mayor De San Marco. Obtenido de https://n9.cl/fef4g

Guevara-Mora, G. (2010). Aprendizaje basado en problemas como técnica didáctica para la enseñanzadel tema de la recursividad [problem based learning as a teaching technique for teaching the theme of recursivity]. InterSedes: Revista de las Sedes Regionales, 142-167. Obtenido de https://n9.cl/e9tp5 
Jaramillo-Naranjo, L., \& Puga-Peña, L. (2016). El pensamiento lógico-abstracto como sustento para potenciar los procesos cognitivos en la educación [Logical-abstract thinking as a support to enhance cognitive processes in education]. Sophia, Colección de Filosofía de la Educación(21). doi:http://dx.doi.org/10.17163/soph.n21.2016.01

Larrazolo, N., Backhoff, E., \& Tirado, F. (2013). Habilidades de razonamiento matemático de estudiantes de educación media superior en México [Mathematical reasoning skills of upper secondary education students in Mexico]. Revista mexicana de investigación educativa, 18(59). Obtenido de https://n9.cl/n0vyx

Leiva-Sánchez, F. (2016). ABP como estrategia para desarrollar el pensamiento lógico matemático en alumnos deeducación [ABP as a strategy to develop mathematical logical thinking in secondary education students] secundaria. Sophia, Colección de Filosofía de la Educación, 209-224. doi:10.17163/soph.n21.2016.09

Matamorros-Espinoza, W. (2018). Propuesta didáctica de Aprendizaje Basado en Problemas dirigida al área de matemáticas ( $8^{\circ}$ de educacióngeneral básica): caso Unidad Educativa "Sagrada Familia" [Didactic Proposal of Problem-Based Learning directed to the area of mathematics (8th of bas]. Quito: Pontificia Universidad Católica Del Ecuador. Obtenido de https://n9.cl/vslr

Mendoza-Novillo, P. A., Erazo-Álvarez, J. C., \& Narváez-Zurita, C. I. (2019). Estudio de tiempos y movimientos de producción para Fratello Vegan Restaurant [Study of production times and movements for Fratello Vegan Restaurant] Recuperado de: https://n9.cl/jbxc. Cienciamatria, 271-297. doi:https://doi.org/10.35381/cm.v5i1.267

Nasution, M., Yerizon, Y., \& Gusmiyanti, R. (2018). Students' Mathematical ProblemSolving AbilitiesThrough The Application of Learning ModelsProblem Based Learning [Habilidades de resolución de problemas matemáticos de los estudiantes mediante la aplicación de modelos de aprendizaje Aprendizaje basado en]. IOP Conference Series: Materials Science and Engineering, 1-6. doi:10.1088/1757$899 X / 335 / 1 / 012117$

Navarro-Casabuena, L. (2017). El pensamiento matemático: una herramienta necesaria en la formación inicial de profesores de matemática [Mathematical thinking: a necessary tool in the initial training of mathematics teachers]. Varona, 1-7. Obtenido de https://n9.cl/mlb9 
Paredes-Curin, C. (2016). Aprendizaje basado en problemas (ABP): Una estrategia de enseñanza de la educaciónambiental, en estudiantes de un liceo municipal de Cañete [Problem-based learning (ABP): A teaching strategy of environmental education, in students of a municipal high]. Revista Electrónica Educare, 1-26. doi:http://dx.doi.org/10.15359/ree.20-1.6

Piaget, J. (1991). Seis Estudios de la Psicología [Six Studies of Psychology]. Barcelona, España: Labor, S.A. Obtenido de https://n9.cl/kyg7

Polya, G. (1965). Cómo plantear y resolver problemas [How to solve it]. Distrito Federal de Mexico: Trillas S.A. Obtenido de https://n9.cl/dx2m

Saltos-Cedeño, A., Vallejo-Valdivieso, P., \& Moya-Martínez, M. (2020). Innovación en educación matemática de básica superior durante el confinamiento por COVID19. [Innovation in higher basic mathematics education during confinement by COVID-19]. KPISTEME KOINONIA, 3(5), 142-161. http://dx.doi.org/10.35381/e.k.v3i5.723

Valderrama-Sanabria, M., \& Castaño-Riobueno, G. (2017). Solucionando dificultades en el aula: una estrategia usando el aprendizaje basado en problemas [Solving difficulties in the classroom: a strategy using problem-based learning]. Cuidarte, 1907-1918. Obtenido de https://n9.cl/6nnso

Vargas, A., \& Labatut-Portilho, E. (2017). Metacognição em Grupos de Problem-based Learning (PBL) [Metacognición de grupos de aprendizaje basados en problemas (PBL)]. Educação. Revista do Centro de Educação, 421-434. doi:https://doi.org/http://dx.doi.org/10.5902/1984644424497 